\title{
A Way to Evaluate the Site Classification by Chinese Seismic Design Specification From VS30data of NGA

\author{
Mei-Li SUN ${ }^{1, *}$ Xia-xin TAO and Rui ZHI ${ }^{2}$
} \\ ${ }^{1}$ Key Laboratory of Earthquake Engineering and Engineering Vibration, Institute of Engineering Mechanics, China Earthquake Administration, Harbin, 150080, China \\ ${ }^{2}$ School of Civil Engineering, Harbin Institute of Technology, Harbin, 150090, China ${ }^{*}$ Corresponding author: E-mail: taoxiaxin@aliyun.com
}

Keywords: Site Classification, Shearwave velocity, VS20, VS30, NGA.

\begin{abstract}
On the basis of Vs30 and Vs20calculated from shearwave velocity profiles of 732 boreholes, a formula to estimate Vs20 from Vs30 is presented in this paper by reference to a recent suggestion, an iterative process to estimate rough overburden thickness is suggested based on two formulas for the average velocity at depth $\mathrm{z}$ from Vs30 and Vs20, and the velocity between two depths. A way to evaluate the site category by Chinese classification from the corresponding Vs30 is worked out.
\end{abstract}

\section{Introduction}

As well known, local site condition influences the ground motion during earthquake significantly, and earthquake action in seismic design code stipulated always with site classification. The basis of the stipulation, strong ground motion data base, must include site information for each record. For example, NGA-West2 database, the largest motion database nowadays in the world, provides sitecategory in $\mathrm{A}, \mathrm{B}, \mathrm{C}, \mathrm{D}, \mathrm{E}$ and $\mathrm{F}$ for each observation station, and each record links with its own station. However, the classifications in codes of main earthquake countries are not same, even quite different. For example, the above A F classification is commonly adopted in codes of the United States, primarilyfromVs30, the average shear wave velocity in ground $30 \mathrm{~m}$ depth[1], while the classification in Chinese seismic design code is in category I, II, III and IV fromVs20 and the overburden thickness[2].Chinese researchers and engineers should know a corresponding relation between the two classifications, in their application of the NGA data base. The shear wave velocity profiles of 732 boreholes are selected from KiK-netin Japan and the databases of strong motion observation stations in California, US, as the data set of this paper. On the basis of Vs30 and Vs20calculated for each borehole site from the data, and the site classification by the codes of the two countries, a way to estimate Vs20 and rough overburden thickness from the correspondingVs30 is presented mainly by reference to a recent suggestion[3].

\section{Site Classifications in Seismic Design Specifications of US and China}

In US seismic design specification, site is classified into six categories, according mainly to average shear wave velocity,Vs30, of the soil and geotechnical characteristics, as listed in Table 1. 
Table 1. Site classification of US code (from [4])

\begin{tabular}{ccc}
\hline $\begin{array}{c}\text { Site } \\
\text { category }\end{array}$ & Rock \& soil type & $V_{s 30}(\mathrm{~m} / \mathrm{s})$ \\
\hline A & Hard rock & $>1500$ \\
B & Rock & $760-1500$ \\
C & Very stiff soil\& soft & $360-760$ \\
D & rock & $180-360$ \\
E & Medium stiff soil & $<180$ \\
F & Medium soft soil & Requiring site-specific \\
& & evaluations \\
\hline
\end{tabular}

The $V_{s 30}$ in the table is calculated by Eq. 1

$$
V_{s 30}=\frac{\sum_{i=1}^{n} d_{i}}{\sum_{i=1}^{n}\left(d_{i} / v_{s i}\right)} .
$$

Where di is depth of the ith soil or rock layer (m), vsi is shear wave velocity of the ithsoil or rock layer $(\mathrm{m} / \mathrm{s})$, nis the total number of the soil or rock layers from ground surface to the depth of 30 meters.

In Chinese seismic design specification, site is classified into four categories, accordingto average shear wave velocityVs20and overburden thickness, as listed in Table 2.

Table 2. Site classification of Chinese code (from [2])

\begin{tabular}{cccccc}
\hline & $\mathrm{I}_{0}$ & $\mathrm{I}_{1}$ & $\mathrm{II}$ & $\mathrm{III}$ & $\mathrm{IV}$ \\
\cline { 2 - 6 } & \multicolumn{5}{c}{ Overburden thickness $(\mathrm{m})$} \\
\hline$V_{s}>800$ & 0 & & & & \\
$800 \geq V_{s}>500$ & & 0 & & & \\
$500 \geq V_{s 20}>250$ & & $<5$ & $\geq 5$ & & \\
$250 \geq V_{s 20}>150$ & & $<3$ & $3-50$ & $>50$ & \\
$V_{s 20} \leq 150$ & & $<3$ & $3-15$ & $15-80$ & $>80$ \\
\hline
\end{tabular}

In the table, categories I0 and I1 are classified from category I further, and Vs20 is calculated by almost the same equation as Eq.1, but the $n$ here is limited to the number of the soil or rock layers within the depth of 20 meters

One can find from the tables that the main differences between the two classifications are: (1) concerned depths of the shear wave velocities are 30 versus 20 meters, (2) categorizing indices are single versus dual. In general, site A and B of US correspond to Chinese site I, site C corresponds to site I and II, site D to site II and III, site $\mathrm{E}$ and $\mathrm{F}$ to site IV.

\section{Relation Between Vs30 and Vs20 Adopted in This Paper}

Based on Boore's work[5], Wang suggested a formula to estimate average shear wave velocity above depth $\mathrm{z}$ from the other two velocities respectively above depthsz 1 and $\mathrm{z} 2$, given $\mathrm{z} 1<\mathrm{z} 2$, as follows[3]. 


$$
\log V_{s z}=\log V_{z}\left(z_{2}\right)+\frac{\log z-\log z_{2}}{\log z_{2}-\log z_{1}}\left[\log V_{z}\left(z_{2}\right)-\log V_{z}\left(z_{1}\right)\right]
$$

From shear wave velocity profiles of 732 boreholes from California of US[6] and KiK-net of Japan[7], an empirical relation between Vs30 and Vs29 is firstly fitted, as following.

$$
V_{s 29}=0.9877 V_{s 30}-0.6848 \text {. }
$$

The comparison of the data points of Vs30 and Vs29 from the line by Eq. (2) is shown in Fig. 1.

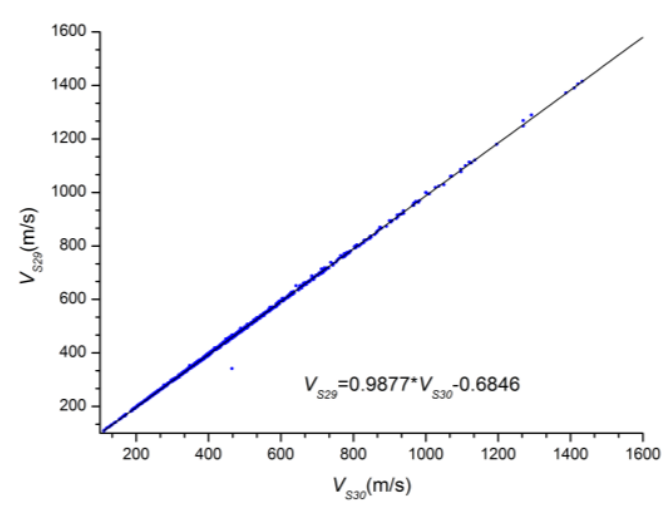

Figure 1. The fitted Vs29-Vs30 relation

Substitute Eq. (3) into Eq. (2), a formula to estimate $V_{\mathrm{s} 20}$ from $V_{\mathrm{s} 30}$ can be obtained as

$$
\log V_{s 20}=\log V_{s 30}-11.9601 \cdot\left[\log V_{s 30}-\log \left(0.9877 V_{s 30}-0.6848\right)\right] .
$$

The values of correlation coefficient and standard deviation of Eq. (4) are 0.9829 and 0.0388 respectively. The distribution of the data points of the Vs30 and Vs20calculated by Eq. (1) directly from the borehole profiles comparing with the line of Eq. (4) is shown in Fig. 1. The dash line in the figure is from Boore's formula[5], for comparison.

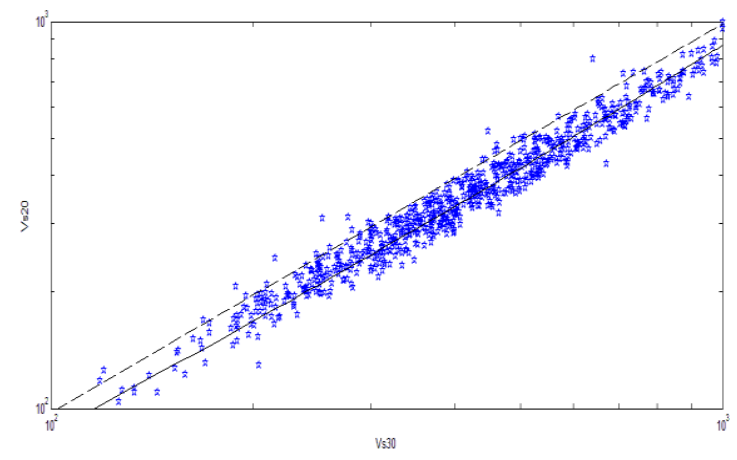

Figure 2. Comparison of the Vs20-Vs30data with the lines by formulas

\section{A Rough Estimation of the Overburden Thickness by Vs30}

The overburden thickness is defined in Chinese code as the minimum depth from ground surface to the top of a stable soil layer with shear wave velocity no less than $500 \mathrm{~m} / \mathrm{s}$ at the site in general. It is impossible in nature to estimate the thickness only from Vs30; fortunately a rough estimation is enough for the site classification from 
the Table 2. A way is suggested here as a searching process, basically by Eq. (1). Taking $20 \mathrm{~m}$ and $30 \mathrm{~m}$ as the $\mathrm{z} 1$ and $\mathrm{z} 2$ in Eq. (1), a formula can be derived for average shear wave velocity above any depthz as

$$
\log V_{s z}=\log V_{s 30}+\frac{\log z-\log 30}{\log 30-\log 20}\left[\log V_{s 30}-\log V_{s 20}\right] \text {. }
$$

The formula to estimate the average velocity Vszm between thedepthsz and $z+1$ must be

$$
V_{s z m}=\frac{(z+1-z) \cdot V_{s z+1}}{z+1-z \cdot V_{s z+1} / V_{S z}}=\frac{V_{s z+1}}{z+1-z \cdot V_{s z+1} / V_{s z}} \text {. }
$$

Firstly taking initialvalue of $\mathrm{z}$ as 1.0 , calculate Vszby Eq. (5)and the overburden thickness must be $1.0 \mathrm{mif}$ Vszis larger than $500 \mathrm{~m} / \mathrm{s}$, or to be estimated in the iterative processelse. Then 1)Calculate Vsz+1by Eq. (5) and Vszm by Eq. (6),2) If Vszm is larger than $500 \mathrm{~m} / \mathrm{s}$ then the overburden thickness must be the value ofz, else to be estimated to the 1) step again with a new value of $z=z+1$. The iterative processis ended whenever the overburden thickness has been determined or the zis larger than the maximum value in the line the Vsz value corresponding to in Table2. IF the estimated overburden thickness is less than $20 \mathrm{~m}$, the value of Vs20 is modified by the corresponding Vsz value, according to Chinese site classification[2]. The site category can be evaluated from the final Vs20 and overburden thickness.

\section{Conclusion}

In order to work out a corresponding relation between the classifications of US and China, shearwave velocity profiles of 732 boreholes are selected. On the basis of Vs30 and Vs20calculated for each borehole site from the data, a formula to estimate Vs20 from Vs30 is presented in this paper by reference to Wang's recent work[3]. An iterative process to estimate rough overburden thickness is furthermore suggested based on two formulas for the average velocity at depth z from Vs30 and Vs20, and the average velocity between two depths. Finally a way to evaluate the site category by Chinese classification from the corresponding Vs30 is worked out.

\section{Acknowledgement}

This work is supported by grant 51678540,51478443 and 51178151 of National Nature Science Foundation of China.

\section{References}

[1] International Code Council. 2012 International Building (2012).

[2] Ministry of housing and urban-rural development of the People's Republic of China, General Administration of Quality Supervision, Inspection and Quarantine of the People's Republic of China. Code for seismic design of buildings (GB500112010).

[3] Wang H Y, S Y Wang. Bulletin of the Seismological Society of America, 105, 1359 (2015). 
[4] Building Seismic Safety Council.2003 Edition NEHRP Recommended Provisions for the Development of Seismic Regulations for New Buildings and Other Structures, FEMA 450, (2004).

[5] Boore D M, E M Thompson, H Cadet. Bulletin of the Seismological Societyof America, 101, 3046 (2011).

[6] Boore D M. Bulletin of the seismological society of America, 94, 591 (2004).

[7] Aoi S, T Kunugi, H Fujiwara. Strong-motion seismograph network operated by NIED: K-NET and KiK-net. Proc. of Japanese conference on earthquake engineering, (2004). 